\title{
Systematic review of the evidence for rational dosing of colistin
}

\author{
E Visser Kift, ${ }^{1} \mathrm{MB}$ ChB, BScMedSc (Hons); G Maartens, ${ }^{1} \mathrm{MB}$ ChB, MMed (Int Med); C Bamford, ${ }^{2} \mathrm{MB}$ ChB, MMed (Med Micro), MPhil \\ ${ }^{1}$ Division of Clinical Pharmacology, Department of Medicine, University of Cape Town, South Africa \\ ${ }^{2}$ Division of Medical Microbiology, Department of Clinical Laboratory Sciences, University of Cape Town, and National Health Laboratory Service, \\ Groote Schuur Hospital, South Africa
}

Corresponding author: G Maartens (gary.maartens@uct.ac.za)

\begin{abstract}
Background. There is an alarming global increase in the incidence of nosocomial infections with multidrug-resistant Gram-negative bacteria, which are often only susceptible to colistin. Colistin was developed prior to current methods of establishing dosing using pharmacokinetic-pharmacodynamic relationships. Dosing regimens differ in package inserts from different manufacturers and in different guidelines. It is imperative to avoid under-dosing with colistin in order to limit the development of resistance, as it is the last line of defence. Methods. We conducted a systematic review of the literature to develop guidelines for rational dosing of intravenous colistin, with a particular focus on critically ill patients.

Results. Colistin is administered as the inactive pro-drug colistimethate sodium. Colistin demonstrates concentration-dependent bacterial killing, suggesting that higher doses should be administered less frequently to achieve higher peak concentrations. Dose-related nephrotoxicity occurs, making it impossible to safely achieve concentrations that prevent the selection of resistant mutants or the effective eradication of bacteria with higher minimum inhibitory concentrations. Theoretically, combination therapy should be used to reduce the risk of selection of resistant bacteria. In critically ill patients, a loading dose should be given to rapidly achieve therapeutic concentrations, followed by maintenance doses of $4.5 \mathrm{MU}$ 12-hourly. Maintenance dose adjustment is necessary with renal impairment.

Conclusion. Easier access to colistin is needed in South Africa, where it is not a registered medicine. Further research is needed to better characterise colistin's pharmacokinetic-pharmacodynamic relationships in humans and to establish whether combinations of colistin with other antimicrobials result in improved clinical outcomes or a reduction in selection of resistant bacteria.
\end{abstract}

S Afr Med J 2014;104(3):183-186. DOI:10.7196/SAMJ.7011

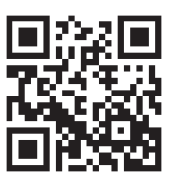

Since the discovery of antimicrobial agents, everevolving mechanisms of microbial resistance have been shaping the field of infectious diseases. Globally multidrug-resistant (MDR) Gram-negative bacilli causing nosocomial infections (notably in Klebsiella pneumoniae, Pseudomonas aeruginosa, Enterobacter sp. and Acinetobacter baumannii) have become an important emerging threat. ${ }^{[1]}$

In South Africa (SA), carbapenem resistance is emerging in $K$. pneumoniae and Enterobacter sp. while high levels of resistance to all antimicrobial classes are observed among $P$. aeruginosa and A. baumannii. ${ }^{[2]}$ A. baumannii is a common pathogen in intensive care units (ICUs). ${ }^{[3]}$ The antimicrobial susceptibility patterns of isolates of bacteraemic A. baumannii complex isolates over time in the public and private sectors in SA are shown in Figs $1 \mathrm{~A}$ and $1 \mathrm{~B}$, respectively. Particularly striking is the decline in carbapenem susceptibility from $35 \%$ in 2007 to only $17 \%$ in 2011 in the public sector. More than half the isolates in the private sector were resistant to carbapenems, but there was no clear downward trend. Inappropriate antibiotic prescription practices in ICUs in the public and private sectors in SA are common and associated with poor patient outcomes. ${ }^{[4]}$ Both carbapenem resistance and inappropriate antibiotic use are associated with increased mortality in patients with Acinetobacter bacteraemia. ${ }^{[5-8]}$

Many acinetobacteria and other MDR Gram-negative bacteria are only susceptible to colistin (polymyxin E). Colistin is not a registered medicine in SA, but it can be obtained via a Medicines and Related Substance Act, Section 21 application to the Medicines Control Council. The polymyxin group of antibiotics was discovered in the $1940 \mathrm{~s},{ }^{[9,10]}$ but their popularity soon faded due to reports of nephrotoxicity and the availability of safer antibiotics in the 1970 s. $^{[11]}$ Important pharmacokinetic and pharmacodynamic data for colistin are lacking, which are necessary for safe and effective dosing, particularly in critically ill patients and those with impaired renal function. ${ }^{[12]}$ No international consensus exists on the correct dose, and dosing units are not standardised. Package insert dosing instructions differ between manufacturers. An additional problem is that prescribing units differ between manufacturers, including mg for colistin base activity (CBA) and colistimethate sodium (CMS), and international units for CMS. This creates confusion among clinicians and complicates interpreting the available literature. The product accessed in SA (Colimycine) is labelled in international units. One million units (MU) of CMS is roughly equivalent to $80 \mathrm{mg}$ of CMS and $30 \mathrm{mg}$ of CBA.

Under-dosing of colistin increases the risk of the development of resistance and hetero-resistance, which is important as colistin is the last line of defence against MDR Gram-negative bacteria. Colistin resistance was first reported in the Czech Republic in 1999 and worldwide reports are accumulating at an alarming rate. ${ }^{[13]}$ Eighteen of 132 (13.6\%) bloodstream isolates of A. baumannii complex were resistant to colistin in Groote Schuur Hospital, SA, during 2011.

\section{Methods}

We conducted a systematic review of the evidence for rational dosing of intravenous (IV) colistin, with a particular focus on patients who are critically ill or have renal impairment. English language, peerreviewed journal publications (predating April 2013) were identified by searching the PubMed database. The search terms included various combinations of the following keywords: polymyxins; 


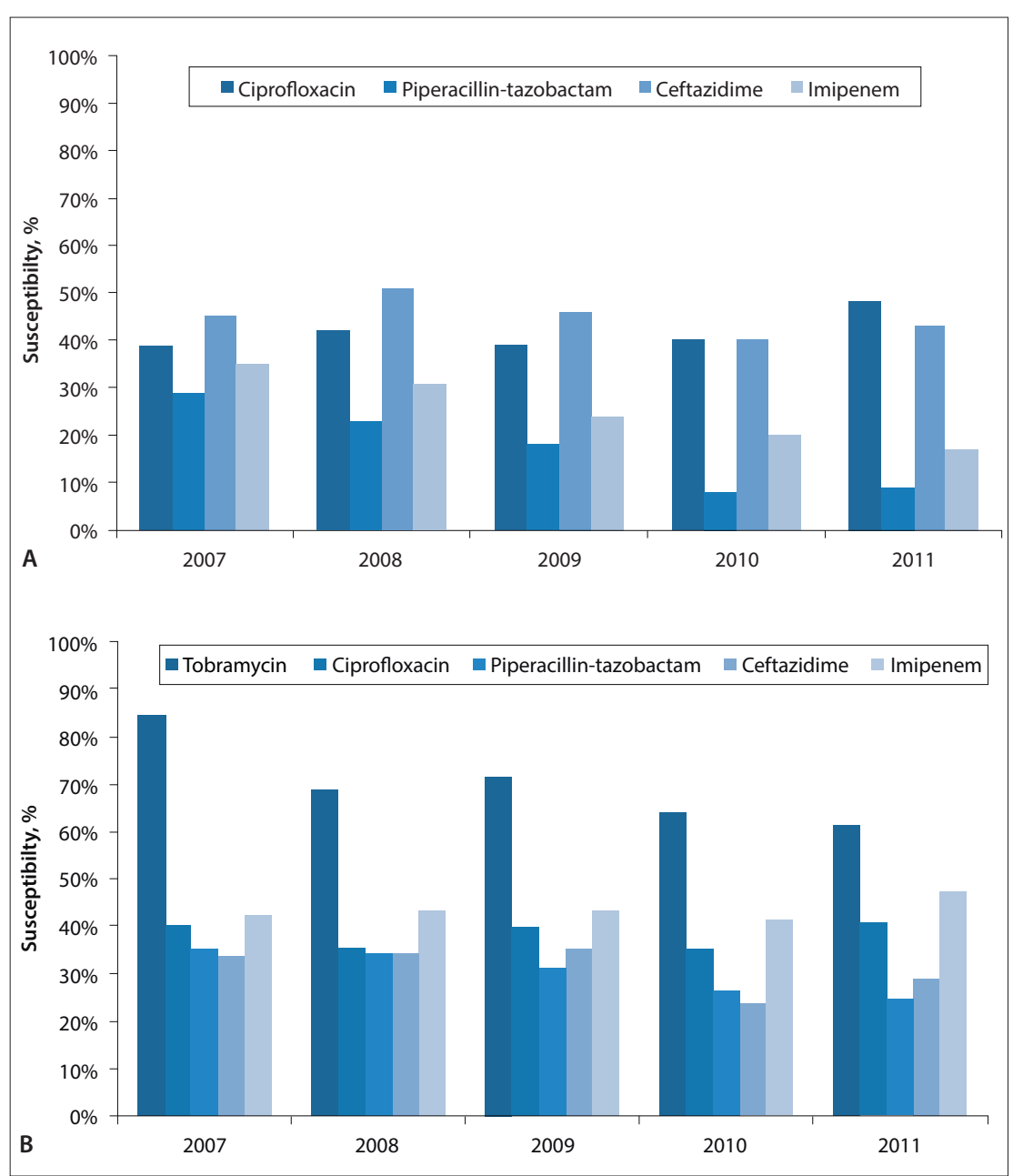

Fig. 1. Susceptibility of A. baumannii complex blood isolates to selected antimicrobial agents at laboratory sites in South Africa, 2007 - 2011. (A) Five large public sector (2011 susceptibility data are based on provisional results); $; 2,43]$ and (B) eight private sector laboratory sites (personal communication, Colleen Bamford for the National Antibiotic Study Forum).

colistin; colistimethate sodium; intravenous; severe sepsis; critically ill; pharmacokinetics; pharmacodynamics; dosing; dosing units; dosing interval; nephrotoxicity; renal failure; renal replacement; resistance; combination therapy; MDR Gram-negative infections; Acinetobacter; Pseudomonas; and Klebsiella. After reviewing the abstracts, relevant fulltext manuscripts were retrieved. Additional articles were identified by hand searching the references of articles obtained by the electronic search strategy. Finally, based on the evidence obtained, an IV colistin dosing guideline for the treatment of MDR Gramnegative infections was developed, with a particular focus on critically ill patients.

\section{Results}

\section{Pharmacokinetic overview}

Colistin is administered intravenously as the inactive pro-drug CMS, which is hydrolysed to active colistin. ${ }^{[14]}$ In critically ill patients, colistin plasma concentrations peak seven hours after CMS administration. The half-lives of CMS and colistin are 2.3 and 14.4 hours, respectively. ${ }^{[15]}$ Distribution to cerebrospinal, pleural and synovial fluid is poor. ${ }^{[10]}$ Unconverted CMS is predominantly eliminated by the kidneys, partly by tubular secretion. By contrast, colistin is predominantly cleared by unknown nonrenal mechanisms and undergoes extensive renal tubular reabsorption. ${ }^{[16]}$

\section{Dose adjustment in renal failure}

In patients with renal impairment the elimination of CMS is decreased and a greater fraction of the administered dose is converted to colistin, ${ }^{[17-19]}$ necessitating a dose adjustment. Both CMS and colistin are efficiently cleared by venovenous haemofiltration ${ }^{[20]}$ and haemodialysis. ${ }^{[17,21-22]}$ Therefore, a supplemental dose of colistin needs to be given after dialysis and higher doses are required in patients undergoing venovenous haemofiltration.

\section{Loading doses}

Drugs take 4 - 5 times their half-lives to reach target steady-state plasma concentrations. A loading dose is required in serious infections to rapidly achieve therapeutic concentrations. Critically ill patients with severe sepsis have significant capillary leak, ${ }^{[32-24]}$ which increases the volume of distribution of colistin 4 - 15-fold. ${ }^{[25-26]}$ The loading dose in critically ill patients is therefore higher than in less-ill patients. ${ }^{[15,17]}$ It is important to note that the magnitude of the loading dose is not affected by renal impairment; only subsequent maintenance doses or dose intervals should be adjusted.

\section{Pharmacokinetic-}

\section{pharmacodynamic relationships}

Colistin has no activity against Gram-positive bacteria and anaerobes, ${ }^{[10]}$ but rapidly kills Gram-negative bacteria in a concentrationdependent manner. ${ }^{[1,1,9,27]}$ The bactericidal activity of colistin is partly due to its detergent effect on the bacterial cell membrane..$^{[10,19,28]}$ This disruptive effect on membrane integrity may account for the in vitro synergy observed with certain antimicrobials (e.g. rifampicin). ${ }^{[28]}$ Murine studies showed that the most predictive index for antibacterial effect against P. aeruginosa and A. baumannii was the ratio of the area under the curve to the minimum inhibitory concentration (AUC/MIC). ${ }^{[29-30]}$

Extrapolating from murine AUC/MIC colistin data, Garonzik et al. ${ }^{[17]}$ estimated that in humans a total colistin AUC/ MIC of 60 is the average achieved using currently recommended doses. This AUC/ MIC of 60 would be expected to result in a suboptimal pharmacodynamic effect of somewhere between stasis and $1 \log _{10}$ kill for most susceptible bacteria. The authors acknowledge that there are limitations to their estimates as free concentrations of colistin were measured in the murine infection models ${ }^{[29-30]}$ and there are no human data on the protein binding of colistin. Peak concentrations of at least $4 \mathrm{mg} / \mathrm{l}$ (four times the MIC) were needed to eliminate $P$. aeruginosa in one study, but in critically ill patients this concentration was only reached with doses of $9 \mathrm{MU}$ of CMS. ${ }^{[31]} \mathrm{A}$ recent in vitro study showed that the mutant prevention concentration (at which $90 \%$ of isolates tested were prevented from developing mutant strains) exceeds $128 \mathrm{mg} / \mathrm{l}^{[32]}$ a concentration not achievable with currently used doses. A high proportion of clinician-selected dosing regimens result in sub-therapeutic colistin concentrations. ${ }^{[12,15,17,26,31,33]}$ Of particular concern is a recent study showing that it is not possible to reach the modest target 
Table 1. IV CMS dosing guideline for the treatment of MDR Gram-negative infections

\begin{tabular}{|c|c|c|}
\hline Dose & Patient category & Dosing suggestion \\
\hline Loading & Critically ill or severe sepsis & $9-12 \mathrm{MU}^{*}$ \\
\hline \multirow[t]{6}{*}{ Maintenance } & $\mathrm{eGFR}>60 \mathrm{ml} / \mathrm{min}$ & 4.5 MU 12-hourly \\
\hline & eGFR $30-60 \mathrm{ml} / \mathrm{min}$ & 3 MU 12-hourly \\
\hline & eGFR $10-30 \mathrm{ml} / \mathrm{min}$ & 2 MU 12-hourly \\
\hline & $\mathrm{eGFR}<10 \mathrm{ml} / \mathrm{min}$ & 1 MU 12-hourly \\
\hline & Intermittent haemodialysis & $\begin{array}{l}1 \mathrm{MU} \text { 12-hourly plus supplemental dose of } \\
1 \mathrm{MU} \text { after each episode of dialysis }\end{array}$ \\
\hline & Continuous renal replacement & 4.5 MU 12-hourly ${ }^{\dagger}$ \\
\hline
\end{tabular}

colistin AUC/MIC of 60 in patients with creatinine clearances $>70 \mathrm{ml} / \mathrm{min}$ without exceeding the upper limit daily dose of $10 \mathrm{MU}$ CMS recommended in the package insert. ${ }^{[17]}$

\section{Dosing interval}

The recommended dosing intervals of colistin range between 6 and 12 hours. The long half-life of colistin suggests that less frequent dosing intervals should be adequate. On theoretical grounds giving higher doses less frequently should result in higher peak concentrations and more effective bacterial eradication. However, extended dosing intervals may lead to periods of low colistin concentrations allowing for resistant subpopulations to occur within a microbial population susceptible to colistin (heteroresistance) ${ }^{[28-30,3]}$ Small, uncontrolled studies report good efficacy, without significant renal toxicity, of high-dose CMS regimens given 12-hourly ${ }^{[35]}$ or daily. ${ }^{[3]]}$ There are no randomised, controlled, clinical trials (RCTs) evaluating the efficacy and safety of once-, twice- and thrice-daily dosing of colistin.

\section{Combination therapy}

A strong theoretical basis exists for the use of colistin as part of combination antimicrobial therapy to maximise antimicrobial activity and to reduce the risk of the emergence of resistance, especially in patients with normal renal function or with bacterial MICs $>1 \mathrm{mg} / \mathrm{l}^{[10,17]}$ As discussed above, currently used doses (9 MU CMS per day in divided doses) can neither achieve the modest target AUC/MIC of 60 in patients with normal renal function, ${ }^{[17]}$ nor prevent the selection of resistant mutant subpopulations. ${ }^{[10,32]}$

Many studies have proposed different colistin combination regimens. The most frequently studied are combinations of colistin with rifampicin or the carbapenems, ${ }^{[13]}$ which both showed synergy in vitro ${ }^{[36]}$ Other in vitro studies report effective combinations with tigecycline, amikacin, fosfomycin, azithromycin, ceftazidime, minocycline and, surprisingly, the glycopeptides vancomycin and teicoplanin. ${ }^{[10,13]}$ Combination with levofloxacin or tobramycin decreased the mutant selection window of colistin. ${ }^{[3]}$ Some in vitro antibiotic combination studies demonstrated bactericidal activity even against colistin-resistant strains and others showed bactericidal activity at sub-MIC concentrations of colistin. ${ }^{[28]}$ There is an urgent need for RCTs comparing the efficacy of different combinations of antimicrobial agents with colistin. One trial showed that the 30-day mortality rate of patients with serious MDR A. baumannii infections was not reduced by the addition of rifampicin to colistin, but the study was limited by the relatively low total daily dose of CMS (6 MU) used. ${ }^{[44]}$

\section{Nephrotoxicity}

In contrast to initial reports that colistin's nephrotoxicity rates approached $50 \%$, the majority of recent studies report much lower renal toxicity rates of $10-30 \%{ }^{[38]}$ A local study found that the risk of nephrotoxicity of colistin (dosed at the relatively low total daily dose of $6 \mathrm{MU}$ of CMS) was similar to that of tobramycin. ${ }^{[39]}$ Colistin's nephrotoxicity is dose-dependent and mostly mild and reversible. ${ }^{[25,38,40]}$ One study showed that rates of nephrotoxicity were significantly higher in patients receiving daily maintenance doses greater than the equivalent of $12 \mathrm{MU}$ of CMS in a $70 \mathrm{~kg}$ patient. ${ }^{[3]}$ Colistin's exact mechanism of nephrotoxicity is not established. ${ }^{[1,1,2,40,41]}$ Patients with abnormal renal function at the start of colistin therapy have consistently been identified as being at high risk for nephrotoxic events. ${ }^{[28]}$ General measures to limit colistin's nephrotoxicity include regular monitoring of renal function with appropriate dose adjustment (especially with prolonged use), adequate hydration and limited use of concomitant nephrotoxic drugs. Therapeutic monitoring of CMS/ colistin concentrations is not commercially available.

\section{Discussion}

It is imperative that colistin is dosed appropriately to minimise the risk of resistance as it is a last-line agent against MDR Gramnegative bacteria, and the pipeline of new drugs in development for these organisms is very small. ${ }^{[42]}$ There are good theoretical grounds to suggest that colistin should be used in combination with other effective antibacterials, especially in patients with normal renal function and when treating bacteria with MICs $>1 \mathrm{mg} / \mathrm{l}^{[10,17]}$

We used the information gleaned from our systematic review to develop simple recommendations for rational dosing (Table 1). A key study that informed our recommendations was conducted by Garonzik et al., ${ }^{[17]}$ who integrated population pharmacokinetic data with pharmacodynamic data in a model to estimate dosing in critically ill patients with a wide range of renal function (including those receiving renal replacement therapy). Even though the maintenance doses we recommend are higher than in the package insert of the currently used unregistered product in SA, these will still only result in concentrations that have suboptimal bacterial killing. Higher doses are likely to result in unacceptable rates of nephrotoxicity. ${ }^{[38]} \mathrm{We}$ suggest 12-hour dosing intervals in view of the long half-life of colistin (14 hours) and because of the theoretical benefit of the resulting higher peak concentrations, but, as discussed above, 8-hourly dosing is also acceptable. CMS is unstable in aqueous solutions, therefore it should be administered shortly after reconstitution. ${ }^{[10]}$ All critically ill patients with severe sepsis, regardless of their renal function, require a loading dose of 9 - $12 \mathrm{MU}$ of CMS to ensure the rapid attainment of 
therapeutic concentrations. ${ }^{[15,17]}$ The loading dose range in the table is based on ideal body weight. The loading dose should be administered intravenously over 1 - 2 hours, followed by the first maintenance dose after 12 hours. ${ }^{[15]}$ Maintenance doses can be given as IV infusions over $15 \mathrm{~min} .{ }^{[2]]}$ In patients with an estimated glomerular filtration rate $<60 \mathrm{ml} / \mathrm{min}$, CMS dose adjustment is required. Twelve-hourly dosing is appropriate in patients with renal impairment, because only CMS, the inactive pro-drug, is subjected to renal elimination (not the active drug colistin). CMS doses equal to or even higher than the daily dose in patients with normal renal function are required in patients on continuous renal replacement therapy, because both CMS and colistin are removed ${ }^{[17,22]}$ Garonzik et al. ${ }^{[17]}$ recommend a daily dose of $16 \mathrm{MU}$ of CMS in this setting.

There is a clear need for further research on colistin, particularly to establish pharmacokinetic-pharmacodynamic relationships in humans. More RCTs need to be conducted to determine if combination therapy results in superior outcomes, and, if so, which combinations should be used. Finally, access to colistin needs to be made easier in SA, especially in the public sector where carbapenem resistance is increasing.

\section{References}

1. Kanj SS, Kanafani ZA. Current concepts in antimicrobial therapy against resistant Gram-negative organisms: Extended-spectrum $\beta$-lactamase-producing Enterobacteriaceae, carbapenem-resistant Enterobacteriaceae, and multidrug-resistant Pseudomonas aeruginosa. Mayo Clin Proc 2011;86(3):250259. [http://dx.doi.org/10.4065/mcp.2010.0674]

2. Bamford C, Bonorchis K, Ryan A, et al. Antimicrobial susceptibility pattern of selected bacteraemia isolates from South African public sector hospitals, 2010. South Afr J Epidemiol Infect 2011;26(4):243-50.

3. Ntusi NB, Badri M, Khalfey H, et al. ICU-associated Acinetobacter baumannii colonisation/infection in a high HIV-prevalence resource-poor setting. PLoS One 2012;7(12):e52452. [http://dx.doi. in a high HIV-prevalence resou

4. Paruk F, Richards G, Scribante J, Bhagwanjee S, Mer M, Perrie H. Antibiotic prescription practices and their relationship to outcome in South Africa: Findings of the prevalence of infection in South African intensive care units (PISA) study. S Afr Med J 2012;102(7):613-616.

5. Kim YJ, Kim SI, Hong KW, Kim YR, Park YJ, Kang MW. Risk factors for mortality in patients with carbapenem-resistant Acinetobacter baumannii bacteremia: Impact of appropriate antimicrobial therapy. J Korean Med Sci 2012;27(5):471-475. [http://dx.doi.org/10.3346/jkms.2012.27.5.471]

6. Kwon KT, Oh WS, Song JH, et al. Impact of imipenem resistance on mortality in patients with Acinetobacter bacteraemia. J Antimicrob Chemother 2007;59(3):525-530. [http://dx.doi.org/10.1093/ $\mathrm{jac} / \mathrm{dk} 1499]$

7. Metan G, Sariguzel F, Sumerkan B. Factors influencing survival in patients with multi-drug-resistant Acinetobacter bacteraemia. Eur J Intern Med 2009;20(5):540-544. [http://dx.doi.org/10.1016/j. ejim.2009.05.005]

8. Lee YT, Kuo SC, Yang SP, et al. Impact of appropriate antimicrobial therapy on mortality associated with Acinetobacter baumannii bacteremia: Relation to severity of infection. Clin Infect Dis 2012:55(2):209Acinetobacter baumannii bacteremia: Relation

9. Li J, Nation R, Milne RW, Turnidge JD, Coulthard K. Evaluation of colistin as an agent against multiresistant Gram-negative bacteria. Int J Antimicrob Agents 2005;25(1):11. [http://dx.doi.org/10.1016/j. ijantimicag.2004.10.001

10. Yahav D, Farbman L, Leibovici L, Paul M. Colistin: New lessons on an old antibiotic. Clin Microbiol Infect 2012;18(1):18-29. [http://dx.doi.org/10.1111/j.1469-0691.2011.03734.x]

1. Nation RL, Li J. Colistin in the 21st century. Current Opin Infect Dis 2009;22(6):535-543. [http:// dx.doi.org/10.1097/QCO.0b013e328332e672

12. Li J, Nation R, Turnridge J, et al. Colistin: The re-emerging antibiotic for multidrug-resistant Gramnegative bacterial infections. Lancet Infect Dis 2006;6(9):589-601. [http://dx.doi.org/10.1016/S14733099(06)70580-1]

13. Cai Y, Chai D, Wang R, Liang B, Bai N. Colistin resistance of Acinetobacter baumannii: Clinical reports, mechanisms and antimicrobial strategies. J Antimicrob Chemother 2012;67(7):1607-1615. [http:// dx.doi.org/10.1093/jac/dks084]

14. Bergen PJ, Li J, Rayner CR, Nation RL. Colistin methanesulfonate is an inactive prodrug of colistin Bergen PJ, Li J, Rayner CR, Nation RL. Colistin methanesulfonate is an inactive prodrug of colistin
against Pseudomonas aeruginosa. Antimicrob Agents Chemother 2006;50(6):1953-1958. [http:// dx.doi.org/10.1128/AAC.00035-06]

15. Plachouras D, Karvanen M, Friberg LE, et al. Population pharmacokinetic analysis of colistin methanesulfonate and colistin after intravenous administration in critically ill patients with infection caused by Gram-negative bacteria. Antimicrob Agents Chemother 2009;53(8):3430-3436. [http:// dx.doi.org/10.1128/AAC.01361-08

16. Li J, Milne RW, Nation RL, Turnidge JD, Smeaton TC, Coulthard K. Use of high-performance liquid chromatography to study the pharmacokinetics of colistin sulfate in rats following intravenou administration. Antimicrob Agents Chemother 2003;47(5):1766-1770. [http://dx.doi.org/10.1128/ AAC. $47.5 .1766-1770.2003$

17. Garonzik SM, Li J, Thamlikitkul V, et al. Population pharmacokinetics of colistin methanesulfonate and formed colistin in critically ill patients from a multicenter study provide dosing suggestions for various categories of patients. Antimicrob Agents Chemother 2011:55(7):3284-3294. [http://dxdol. org/10.1128/AAC.01733-10]
18. Michalopoulos AS, Karatza DC, Gregorakos L. Pharmacokinetic evaluation of colistin sodium. Expert Opin Drug Metab Toxicol 2011;7(2):245-255. [http://dx.doi.org/10.1517/17425255.2011.541439]

19. Michalopoulos AS, Falagas ME. Colistin: Recent data on pharmacodynamics properties and clinical efficacy in critically ill patients. Ann Intensive Care 2011;1(1):30. [http://dx.doi.org/10.1186/21105820-1-30]

20. Marchand S, Frat JP, Petitpas F, et al. Removal of colistin during intermittent haemodialysis in two critically ill patients. J Antimicrob Chemother 2010;65:1836-1837. [http://dx.doi.org/10.1093/jac/ dkq185]

21. Li J, Rayner CR, Nation RL, et al. Pharmacokinetics of colistin methanosulfonate and colistin in a critically ill patient receiving continuous venovenous hemodialfiltration. Antimicrob Agents Chemother 2005;49:4814-4815. [http://dx.doi.org/10.1128/AAC.49.11.4814-4815.2005]

22. Karvanen M, Plachouras D, Friberg LE, et al. Colistin methanesulfonate and colistin pharmacokinetics in critically ill patients receiving continuous venovenous hemodiafiltration. Antimicrob Agents Chemother 2013;57(1):668-671. [http://dx.doi.org/10.1128/AAC.00985-12]

23. Roberts JA, Lipman J. Antibacterial dosing in intensive care: Pharmacokinetics, degree of disease and pharmacodynamics of sepsis. Clin Pharmacokinet 2006;45(8):755-773. [http://dx.doi. org/10.2165/00003088-200645080-00001]

24. De Paepe P, Belpaire FM, Buylaert WA. Pharmacokinetic and pharmacodynamic considerations when treating patients with sepsis and septic shock. Clin Pharmacokinet 2002;41(14):1135-1151. [http:// dx.doi.org/10.2165/00003088-200241140-00002

25. Varghese JM, Roberts JA, Lipman J. Antimicrobial pharmacokinetic and pharmacodynamic issues in the critically ill with severe sepsis and septic shock. Crit Care Clin 2011;27(1):19-34. [http://dx.doi. org/10.1016/j.ccc.2010.09.006]

26. Couet W, Gregoire N, Marchand S, Mimoz O. Colistin pharmacokinetics: The fog is lifting. Clin Microbiol Infect 2012;18(1):30-39. [http://dx.doi.org/10.1111/j.1469-0691.2011.03667.x]

27. Bergen PJ, Bulitta JB, Forrest A, Tsuji BT, Li J, Nation RL. Pharmacokinetic/pharmacodynamic investigation of colistin against Pseudomonas aeruginosa using an in vitro model. Antimicrob Agents Chemother 2010 Sep;54(9):3783-3789. [http://dx.doi.org/10.1128/AAC.00903-09]

28. Landman D, Georgescu C, Martin DA, Quale J. Polymyxins revisited. Clin Microbiol Rev 2008;21(3):449-465. [http://dx.doi.org/10.1128/CMR.00006-08]

29. Dudhani RV, Turnidge JD, Coulthard K, et al. Elucidation of the pharmacokinetic/pharmacodynamic determinant of colistin activity against Pseudomonas aeruginosa in murine thigh and lung infection models. Antimicrob Agents Chemother 2010;54(3):1117-1124. [http://dx.doi.org/10.1128/ AAC.01114-09]

30. Dudhani RV, Turnidge JD, Nation RL, Li J. fAUC/MIC is the most predictive pharmacokinetic/ pharmacodynamic index of colistin against Acinetobacter baumannii in murine thigh and lung infection models. J Antimicrob Chemother 2010;65(9):1984-1990. [http://dx.doi.org/10.1093/jac/ dkq226]

31. Daikos GL, Skiada A, Pavleas J, et al. Serum bactericidal activity of three different dosing regimens of colistin with implications for optimum clinical use. J Chemother 2010;22(3):175-178.

32. Cai Y, Li R, Liang B, Bai N, Liu Y, Wang R. In vitro antimicrobial activity and mutant prevention concentration of colistin against Acinetobacter baumannii. Antimicrob Agents Chemother 2010;54(9):3998-3999. [http://dx.doi.org/10.1128/AAC.00264-10]

33. Markou S, Markantonis SL, Dimitrakis E, et al. Colistin serum concentrations after intravenous administration in critically ill patients with serious multidrug-resistant, Gram-negative bacilli infections: A prospective, open-label, uncontrolled study. Clin Infect Dis 2008;30:143. [http://dx.doi, org/10.1016/j.clinthera.2008.01.015

34. Bergen PJ, Li J, Nation RL, Turnidge JD, Coulthard K, Milne RW. Comparison of once-, twiceand thrice-daily dosing of colistin on antibacterial effect and emergence of resistance: Studies with Pseudomonas aeruginosa in an in vitro pharmacodynamic model. J Antimicrob Chemother 2008;61(3):636-642. [http://dx.doi.org/10.1093/jac/dkm511]

35. Dalfino L, Puntillo F, Mosca A, et al. High-dose, extended-interval colistin administration in critically ill patients: Is this the right dosing strategy? A preliminary study. Clin Infect Dis 2012;54(12):17201726. [http://dx.doi.org/10.1093/cid/cis286]

36. Rodriguez $\mathrm{CH}$, De Ambrosio A, Bajuk M, et al. In vitro antimicrobials activity against endemic Acinetobacter baumannii multiresistant clones. J Infect Dev Ctries 2010;4(3):164-167. [http://dx.doi. org $/ 10.3855 /$ jidc.604

37. Cai Y, Yang J, Kan Q, et al. Mutant prevention concentration of colistin alone and in combination with levofloxacin or tobramycin against multidrug-resistant Acinetobacter baumannii. Int J Antimicrob Agents 2012;40(5):477-478. [http://dx.doi.org/10.1016/j.jiantimicag.2012.06.018]

38. Pogue JM, Lee J, Marchaim D, et al. Incidence of and risk factors for colistin-associated nephrotoxicity in a large academic health system. Clin Infect Dis 2011;53(9):879-884. [http://dx.doi.org/10.1093/cid/ cir611

39. Gounden R, Bamford C, Van Zyl-Smit R, Cohen K, Maartens G. Safety and effectiveness of colistin compared with tobramycin for multi-drug resistant Acinetobacter baumannii infections. BMC Infect Dis 2009:9(1):26. [http://dx.doi.org/10.1186/1471-2334-9-26]

40. Spapen H, Jacobs R, Van Gorp V, Troubleyn J, Honore PM. Renal and neurological side effects of colistin in critically ill patients. Ann Intensive Care 2011;1:14. [http://dx.doi.org/10.1186/21105820-1-14]

41. Hartzell JD, Neff R, Ake J, et al. Nephrotoxicity associated with intravenous colistin (colistimethate sodium) treatment at a tertiary care medical center. Clin Infect Dis 2009;48:1724-1728. [http://dx.doi. org $/ 10.1086 / 599225$

42. Boucher HW, Talbot GH, Bradley JS, et al. Bad bugs, no drugs: No ESKAPE! An update from the Infectious Diseases Society of America. Clin Infect Dis 2009;48(1):1-12. [http://dx.doi. org/10.1086/595011]

43. Bamford C, Badenhorst L, Duse A, et al. Antimicrobial susceptibility patterns of selected invasive pathogens from public sector hospitals in South Africa, in 2007. South Afr J Epidemiol Infect 2009; 24(2):28-30.

44. Durante-Mangoni E, Signoriello G, Andini R, et al. Colistin and rifampicin compared with colistin alone for the treatment of serious infections due to extensively drug-resistant Acinetobacter baumannii. A multicentre randomised clinical trial. Clin Infect Dis 2013:57(3):349-358. [http//dx.doi. org/10.1093/cid/cit253

Accepted 16 September 2013 